\title{
Kinematics of early-type galaxies in compact groups: HCG 67, HCG 74 and HCG 79
}

\author{
P. Bonfanti ${ }^{1}$, F. Simien ${ }^{2}$, R. Rampazzo ${ }^{1}$ and Ph. Prugniel ${ }^{2}$
}

\begin{abstract}
We present measurements of stellar kinematics for seven early-type galaxies in HCG 67, HCG 74 and HCG 79 . These data are aimed at studying the relation between the environment and the dynamics, structure and stellar content of early-type galaxies. In the present three groups, the kinematic features we observed cannot be associated unambiguously to physical interactions. Visible morphological peculiarities do not appear correlated with kinematical perturbations.
\end{abstract}

\section{Introduction}

Several studies show that single galaxies in HCGs show interaction signatures both in their morphology (see, e.g., Mendes de Oliveira \& Hickson 1994) and kinematic properties (Rubin et al. 1991), although this is not in general true for all member galaxies and all groups.

The effects of interactions on early-type galaxies are more subtle as no longliving self-gravitating features (tidal tails) may be produced. These galaxies can be expected to display shells or ripples, distorted isophotes (Davoust \& Prugniel 1988) or transient features in their kinematic profiles (Bonfanti et al 1995; Combes et al 1995). The difficulty of connecting particular kinematic characteristics to interactions has been emphasized by Rampazzo et al. (1998). HCGs are adequate places to study the effect of the environment on the physics of early-type galaxies both from a statistical approach (Zepf \& Whitmore 1990) and from a detailed analysis.

We summarize here our search for such signatures in the early-type galaxies in three systems: HCG 67, HCG 74, and HCG 79 (better known as Seyfert's Sextet).

\section{The Data: Broad-Band Images and Spectroscopic Observations}

We have used high resolution $R$-band frames, kindly communicated to us by Daniela Bettoni (for HCG 67) obtained at the Asiago Observatory, and by Paul Hickson (for all three groups) obtained at CFHT.

Long-slit spectra have been obtained at the 1.93-m telescope of the Observatoire de Haute-Provence, equipped with the CARELEC spectrograph. Spectra

\footnotetext{
${ }^{1}$ Osservatorio Astronomico di Brera, Via Brera 28, I-20121 Milano, Italy

${ }^{2}$ CRAL-Observatoire de Lyon (CNRS: UMR 142), F-69561 St-Genis-Laval cedex, France
} 


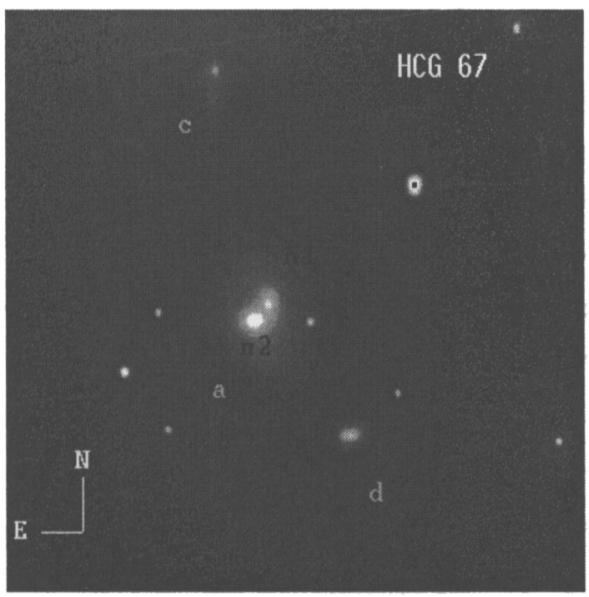

Figure 1. R-band image of HCG 67 with the two nuclei indicated $(\mathrm{n} 1, \mathrm{n} 2)$.

have a dispersion of $66 \AA \mathrm{mm}^{-1}$ corresponding to $1.78 \AA \mathrm{pix}^{-1}$ on the $512 \times 512$, $27 \mu \mathrm{m}^{2} \mathrm{TeK}$ CCD; the actual spectral resolution is $3.2 \AA$. The wavelength range covered is $\approx 4800<\lambda<5750 \AA$. The scale perpendicular to the dispersion is $1^{\prime \prime} 1$, the slit width was 2 "' 1 . K0 III stars were also observed, as templates.

Table 1 summarises the main kinematic parameters while in Figures 1, 2 and 3 examples of the structural and kinematic analysis we performed on the data are shown.

Table 1. Main kinematic parameters from this study

\begin{tabular}{lll}
$\begin{array}{l}\text { Member } \\
\text { ident. }\end{array}$ & $\begin{array}{l}V_{0, \text { hel }} \\
\left(\mathrm{km} \mathrm{s}^{-1}\right)\end{array}$ & $\begin{array}{l}\sigma_{0} \\
\left(\mathrm{~km} \mathrm{~s}^{-1}\right)\end{array}$ \\
\hline HCG 67a & $7246 \pm 24$ & $291 \pm 23$ \\
HCG 67d & $7247 \pm 36$ & $112 \pm 49$ \\
& & \\
HCG 74a & $12208 \pm 32$ & $312 \pm 31$ \\
HCG 74b & $11976 \pm 21$ & $212 \pm 21$ \\
& & \\
HCG 79a & $4237 \pm 18$ & $155 \pm 16$ \\
HCG 79b & $4447 \pm 11$ & $130 \pm 13$ \\
HCG 79c & $4053 \pm 8$ & $59 \pm 20$ \\
\hline
\end{tabular}

\section{Summary and discussion}

Stellar kinematics are seldom available for members of Hickson's compact groups, and our modest sample already represents a substantial fraction of the amount available in the literature. All the groups examined display very compact config- 

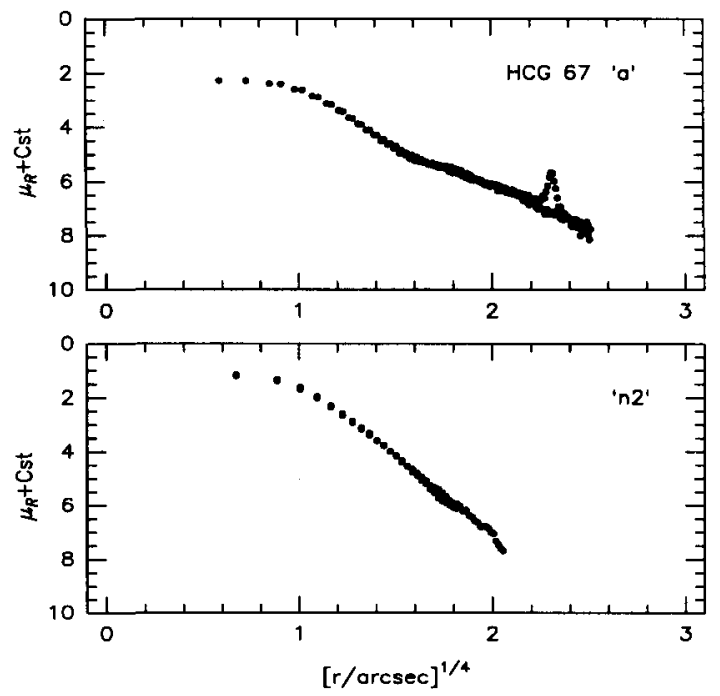

Figure 2. R-band photometric profile of the two nuclei HCG 67a, 'n1' the galaxy itself, and ' 22 ' (actually another galaxy).
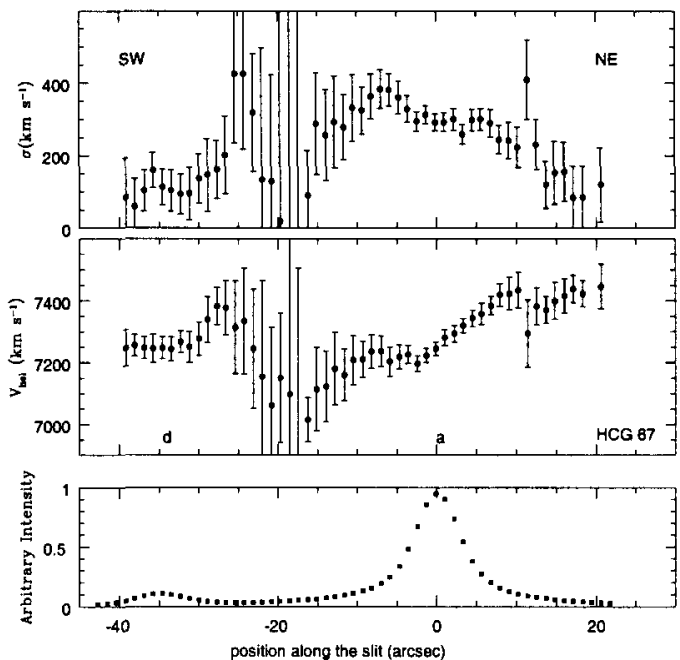

Figure 3. Velocity dispersion, $\sigma$, velocity and relative intensity profiles along the line connecting HCG $67 \mathrm{a}$ and HCG $67 \mathrm{~d}$. 
urations in the sky, are mainly composed of early-type galaxies and, according to the literature, show several morphological signature of interaction. One of them, HCG 67, is embedded within an X-ray diffuse background while HCG 74 and HCG 79 have only an upper limit measured (Ponman et al. 1996).

We have looked for perturbations in the kinematical profiles, and we have carefully examined broad-band images in search of morphological peculiarities which could possibly be associated with these perturbations. In this task, we have attributed more weight to the shape of the rotation-velocity curve than to the velocity-dispersion one, as the former is more likely to be more unambiguously connected to gravitational interactions (Combes et al. 1995). Our measurements show several cases of apparent kinematical interactions, but for all of them, the mere optical superposition cannot be ruled out as the explanation.

Morphological evidence of physical interaction is sometimes elusive, and, for example, the reality of multiple nuclei also suffers from the ambiguity of the position along the same line of sight. For HCG 67, we have been able to show that the putative second nucleus is actually another, smaller galaxy; there is no compelling evidence that it is undergoing a merging process. The fine structure of the central galaxy is, for this group, the strongest optical indication of a connection with the neighborhood. A convincing signature of severe gravitational perturbation is provided by warped disks and irregular extensions, but they cannot be considered as more than mild evidence of interaction within a physical group.

Rampazzo et al. (1998) noticed that morphological signature, kinematical features and the presence of hot gas are poorly correlated with one another. This also emerges from the present study. Limiting ourselves, from now on, to groups showing diffuse X-ray emission (among the more promising argument for physical association together with HI observations), we intend to gather spectroscopic and photometric data in compacts systems and looser ones, where superposition effects would be less severe. We plan to pay attention, again, to kinematical signatures if they appear unambiguous, but also to scaling relations which would link together several photometric and dynamical aspects. For this, a significant sample is needed.

\section{References}

Bonfanti, P. P., Rampazzo, R., Combes, F., Prugniel, Ph., Sulentic J. W. 1995, A\&A 297, 28

Combes, F., Rampazzo, R., Bonfanti, P. P., Prugniel, Ph., Sulentic, J.W. 1995, A\&A, 297, 37

Davoust, E., Prugniel, Ph. 1988, A\&A, 201, 30

Mendes de Oliveira, C., Hickson, P. 1994, ApJ, 427, 684.

Ponman, T. J., Bourner, P. D. J., Ebeling, H., Böhringer, H. 1996, MNRAS, 283,690

Rampazzo, R., Covino, S., Trinchieri, G. Reduzzi, L. 1998, A\&A 330, 423

Rubin, V. C., Ford, W. K., Hunter, D. A. 1991, ApJS, 76, 153

Zepf, S. E., Whitmore, B. C. 1990, ApJ, 418, 72 